\title{
General QED/QCD Aspects of Simple Systems*
}

\author{
Valentine L. Telegd \\ Institute for High Encrgy Physics, ETH. CH-809:. Zumch. Sumtzerland \\ in collaboration with \\ STANLEY J. BRODSkY"* \\ Stanford Linear Accelerator Center. \\ Stanford liniversity. Stanford, California 94909. IS.4 \\ Presented by VLT at the Symposium on the Hydrogen Atom \\ Pisa. Ila!!. June 30 - Ju!y 2. 1988
}

\section{DISCLAIMER}

\begin{abstract}
This report was prepared an an scoount of work aponsored by an apeacy of the Uaited Stales Governmeat. Neither the Unitod Stutes Goverameat nor any uency Uhereof, nor any of their employect, make any warraty, express or implied, or anumes any kged liability or repponsbility for the serurecy, completenes, or usefuleses of any information, opperstur product, of process disklowed, or repreaents that jis use would wok infriage privatedy owned rights. Refer-

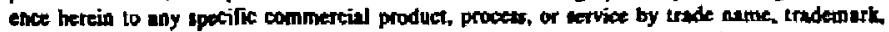
manufecturer, or aberwie does not necenarily cometitute or imply its endorsement, recommendution, of favoring by the Unitod Statcr Government of any usency therool. The views and opiniens of subers expresed herein de not necesussily stale or seflect those of the United Staves Gowernment or any agency thereor.
\end{abstract}

* Work supported by the Department of Energy. contract - Recipient, L.S. Senior Scientist Award of the Alexander ron Humboldt
Foundation. 
Valentine I. Melegii

Institute for High Dergy Fiysics, Ext, Ch-8092 zurich

in collaboration with

Stanley J. Brodsky,

Stanford Iinear Acoelerator Oenter (STAC), Stanford Univ., Stanford, CA 94305

(anrently Aubolat Fellow, MPI Beidelberg)

(presented by VIT)

\section{Bringing you us to date}

The honor of adressing this gathering of distinguished atomic physicists came to one of us (VLT) as a shocking suprise. It is true that quite some time in the past VIT too was a member of the "Inverse Millionaires Club" - that circle of people who masure things to a fraction of a pon - but that was so long ago that it could hardly justify my talking to you now. For a wile VIr thought that the invitation was prompted by his fluency Ir: Italian, but that turned out to be wrong, since the talks are to be given in Dnglish (presumably largely bcoken).

The shock of the irvitation becane even greater when VLT saw the title proposed for his talk: "Ceneral Gantum Electrodynamic Aspects Related to the Spectroscogy of simple Atonic Systems". Only a comittee of seascred sadists could assign such - subject to an experimental physicist, and only an inveterate mascohist could volunteer to acoept it Very fortumately the printed program had a vague title: "Ceneral Quantum Electrodynamic Aspects", but even that sounded like an ifmossible challenge.

Under these circunstances, after having foolishly accepted (who can resist a chance to see Pisa again?), VIT decided on the following strategem: a) change the title so as to bring this audienoe up to date on sone modern topics less familiar to this audience than the cre proposed, b) get himself a collaborator with impeccable credentials. Stan Brodsky has kindly agreed to assist VIr in an otherwise inposible task.

Paraphrasing what has been said of the fanous treatise by Landau and Iifshitz, one could say "inis talk w11 not contain a single formula by relegdi, and not a single word by Erodsky".

This Conference is devoted to the Hydrogen Aton and its younger relatives like positronium. The latter, composed of (presumbly) point-1ike objects, is the ideal testing ground for aD. It should hence be of interest to this audience to be ceminded of the fact that the last decade has led to the discovery and detasled ctudy of now bound partiole-antiparticle systens, which we shall guarkonia, since 
they consist of bound quark-antiquark pairs. There can in principle be as many guch systems as there are "flavors" of quarks (e.g. s, c, b ...) in increasing order of heavyness). The nost interesting ones of these are "chamonium" $(\alpha \bar{c})$ and "bottomiun" (b5), since for these havy quarks a nan-relativistic description is quite adeguate, $\left(m_{c}-1.5 \mathrm{GeV}, m_{b}=5 \mathrm{GeV}\right.$; it is amsing to note that the ground itate of bottomium has about $10^{4}$ tines the dass of positronima). .

Figures 1 and 2 show, respectively, the presently well established levels of chamonium and bottonilm. Today more levels are knom for these systems than for positronium, and more "spectral lines" (transitions) have been identified than were known for hydrogen in Ralmer's days!

What is most remaricable about these levels? Probably two facts: first, although they are hadronic states, they are lang-lived; electromagnetic transitions (E1) compete in general appreciably with the emission of mesons. Second, there is really no "series limit" in the sense of ionization into $Q+\overline{0}(Q=c$ or $b)$.

The $Y$ and $Y$ states are fomed as sharp reomanoes in ê collisions. This identifies their spin (J), parity (P) and charge conjugation (C) quantun numbers readily as those of the photon: $J^{P C}=1^{-}$. The quantum nubers of the states are readily assigned by using rell-known (e.m. and hadronic) selection rules. This results in the $S^{P C}$ values given at the botton of Figs 1 and 2.

From a certain excitation on, the $Y$ (and $M$ ) states can disscciate into two charge-conjuggate mesons $M, \bar{M}$ acoording to the schene

$$
\bar{\alpha} \rightarrow \bar{\alpha}+\bar{Q} \bar{g}=M+\bar{M}
$$

where $g$ is a very light quark ( $d$ ar $u$ ). The combination ( $\alpha \bar{q}$ ) is called a D-meson, the combination (baj) a B-mescn. The corresponding thresholds are indicated in the Figs. by shaded bands. Above these, "hidien cham" turns into "open charm", "hidden beauty" into "open beauty". (The reason for a new nane for the flavor "b" should be obvious.) After all the $J^{P C}$ assigments are made, one can - within the framework of the "naive" quarkoniun model - assign the standard spectroscopic labels to the levels. This is show in the overlay. The standard $n=1$ and $n=2$ posityonium levels appear, but in adation morny excited 's, states. The spacing of the latter indicates that the effective potential (if there is anel) is much eoftar than the faniliar $1 / r$.

Many authors have proposed phenomenological potentials which yield all the cheerved states, and predict new ones (e.g. D states) yet to be discovered. The cocresponding wave functions yield $E 1$ matrix elements in reasonable agreenent with experiment.

The task is to prodict the "observed" potentials from first principles. The current theory of strong interactions, guantum chromo-dynamics (COD), gualitativeIy succeeds in achleving this. This gauge theory patterned after $O \equiv D$ is believed 


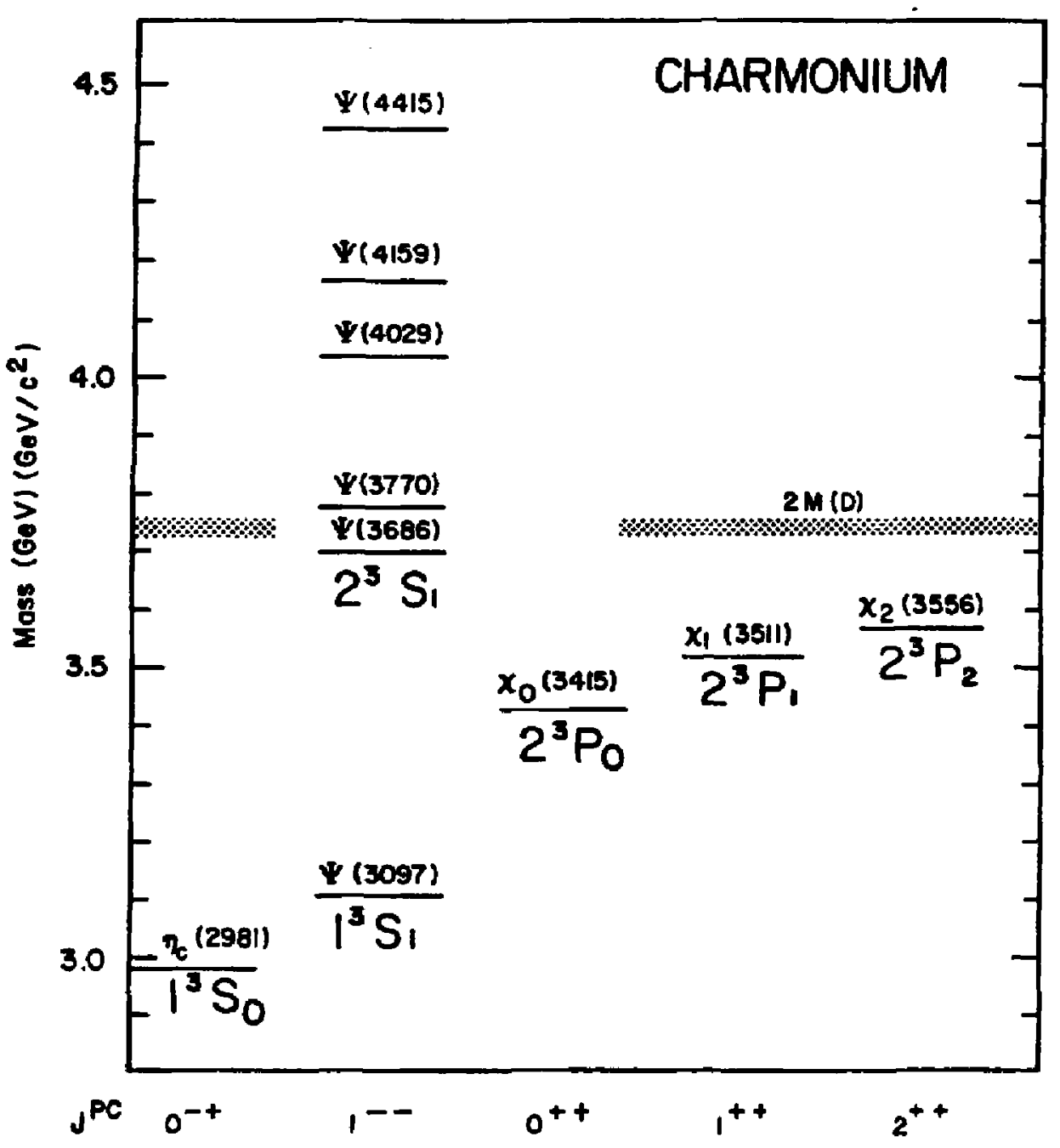

Chamonium $(\alpha \overline{)})$ spectrum. The band at mass = 2u(D) denotes the flavor threshold, wove which levels are broadar than those below $1 t$.

Fig. 1 


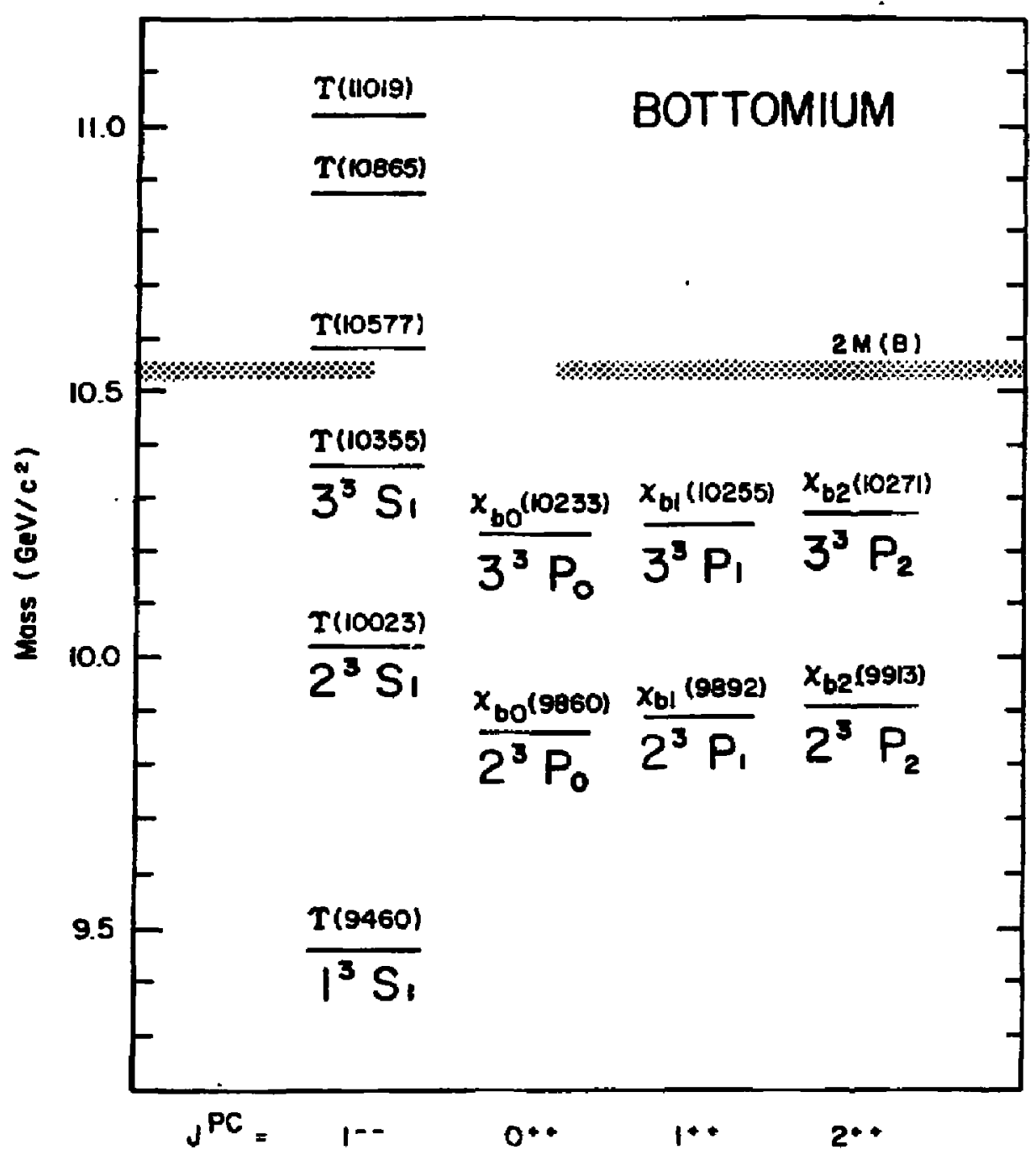

Spectrum of the upsilon ( $(5)$ ) family. Levels ahove flavor threshold Iband at mass - $2 M(B)]$ ate broader than levels below it.

Fig. 2 
to explain why there is no series linit for quarkonia: quarks are forever "confined" within any hadron. It also explains why the guarkonium states are so narrow. In strict analogy with positronium, the $C=-1$ states can go only into three, the $C=+1$ states only into two $C=-1$ field gunta (called gluons). Indeed the $\chi\left(x^{3} P\right)$ states are cberved to be wider than the $Y$ or $r\left({ }^{\prime} s_{1}\right)$ states. We shall return to the COD-ODD analogies Later.

Another novelty wich deserves your attention is the nature of the beloved fine-structure constant a. It is, as we shall discuss later in more detail, a "constant" anly in processes imolving very small momentum transfers.

Next, and more importantly, there is the fact that 030 has become but part of a broader gauge theory wich includes "reak" interactions. Through the discovery of the heavy vector bosons $z^{\circ}$ and $\mathrm{w}^{*}$ at Copv this theory has been brilliantly confimed. The photon's heavy partner, the $z$. Is exchanged between essentially aIl particles, not only the charged ones. Atomic parity violation experiments have confirmed this: Iaporte's rule is daad. The "heak" analogs of a are also energy dependent, so that at some point the "weak" and electronagmetic forces become comparable, whereby the term "weak" loses its maaning. This is illustrated in Fig. 3.

The coupling constant of the strong interaction $(\alpha O D), \alpha_{s}$, decreases with increasing momentum transfer - a point we shall discuss in detail loter. There have been proposals for a Grand Unified (gauge) Theory, GJT, where all three Interactions become equally "strang" at same very high energy. This is also Indicated in Fig. 3.

QED is the model gauge theory after which all others are patterned. We shall divide the discussion of its current status into two parts: closed subjects, and open suijects. To chese one may refer respectively as the "nug" and the "dirt", recalling Feyman's famous statement that he got the Nobel Prize for being better than others in sweeping the dirt under the rug.

\section{2. "Clooed" subjects (the "2uan")}

Q:50, which is supposed to provide finite answers to all orders of pertubation theory (ADPT), can be represented as resting on a foundation (local gauge invariance) and on three pillars (aee Fig. 1). Iocal gauge invariance inplies that the theory is invariant under arbitrary phase transformations of the electron field at anch point in space and tine. The generalization of this principle to invariance under unitary matrix transfomations of the fermion flelds leads to the concept of non-abelian gauge theories which include guantun chromodymamics and the unified electrowak theory. The three pillars are: 


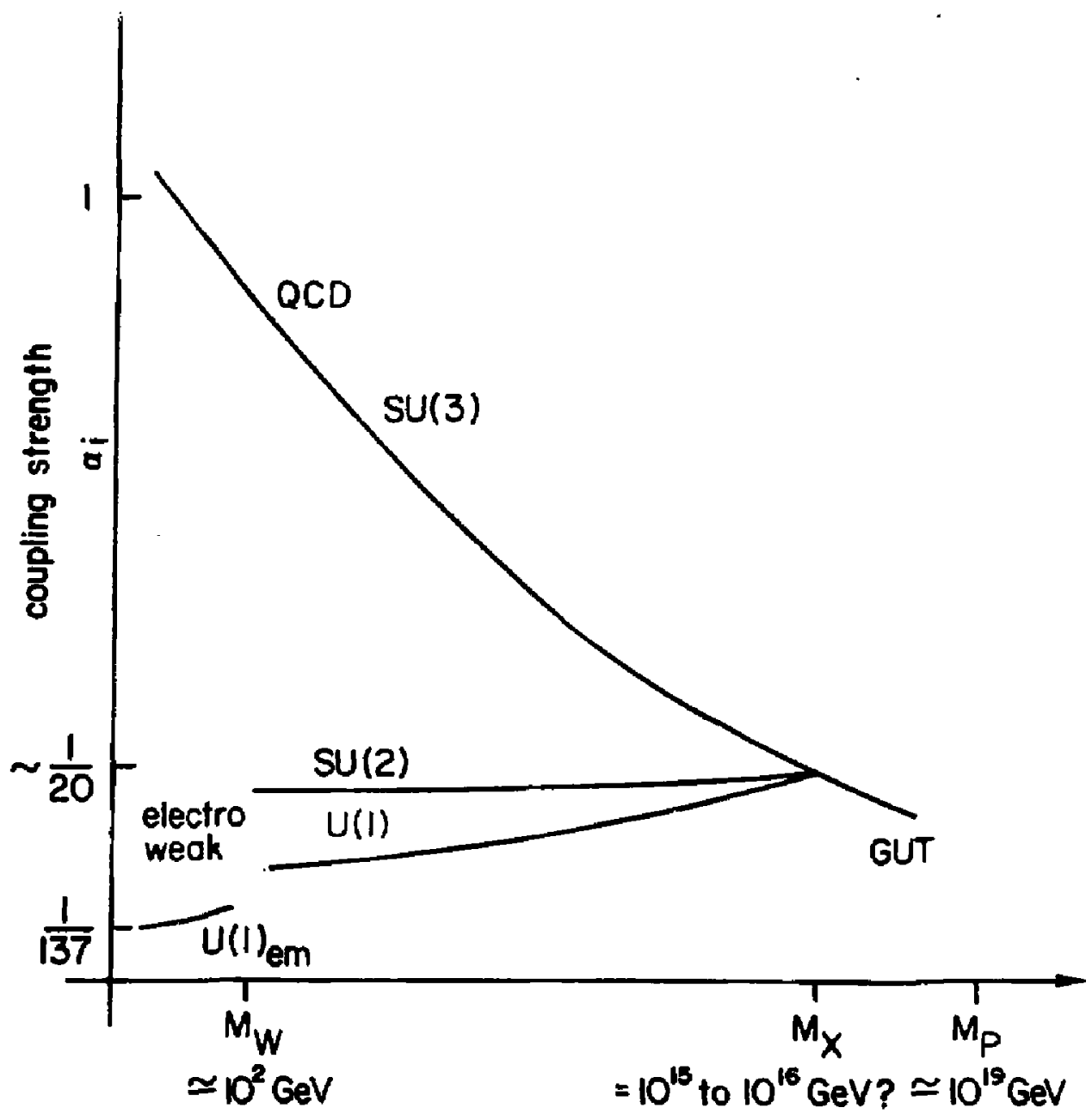

fig. 3 


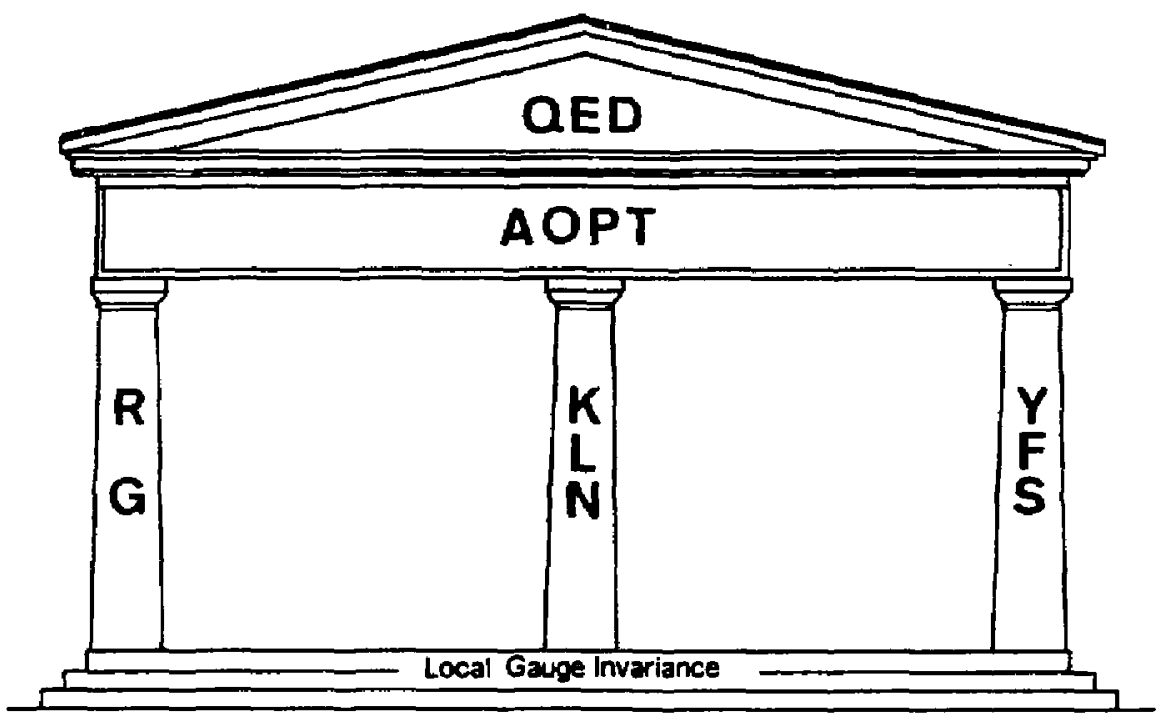

Fig. 4 
2.1 Renomalization theory, and in particular the treatment in terms of the renorwalization group. The Latter goes back to an idea of Petemann and stueckelberg, and was formulated guantitatively by cell-tann and Low. The essenve is that only the cobserved mass $m$ and the observai charge $e$ of the election (and/or its heavier brother leptons $M$ and $t$ ) enter into the final results. vitraviolet infinities (k +- ) are consistently eliminated to AOPT. The compling is characterized by a "running" copling constant which incorporates vacurm polarization to all oriers, viz.

$$
\varepsilon_{r}\left(Q^{2}\right)=\frac{\pi\left(Q_{0}^{2}\right)}{1-\pi\left(Q^{2} / Q_{0}^{2}\right)}
$$

where $Q=\left(4-\right.$ momentum) of interest, and $Q_{0}$ a "reference" 4-womentum. The function It is given by

$$
\pi=\frac{\alpha\left(Q_{0}^{2}\right)}{3 \pi} \ln \left(Q^{2} / Q_{0}^{2}\right)+\ldots
$$

where both $Q_{0}^{2}$ and $Q^{2} \gg m_{1}^{2}$ (lepton mass). Reinterpreting things in coordinate space, (1) simply means that the effective coupling decreases with increasing distance: one observes the shielding due to virtual pairs. At extremely small distances where $K\left(Q^{2}\right)$ is of oxder 1 , i.e., $10^{-21} \mathrm{~cm}$, one could have a blow-up ("Landau singularity") where the theory beomes undefined; this may however be "cured" by the unification of $0 \mathrm{DD}$ with other interactions.

The current, rather successful, theory of strong interactions, $\infty$, is patterned after 000 . It is a scenario there quarks play the rale of leptons, massless vector gluons the part of the photon (gauge bosons), and "color" that of the change. The big difference with electromagnetism is that both the souroes (quarks) and the fields (gluons) carry color, i.e. charge. One is again led to running oopling constant anilogous to ${ }^{\prime}$, viz.

$$
\begin{aligned}
a_{s}\left(Q^{2}\right)=\frac{a_{s}\left(Q_{0}^{2}\right)}{1-\pi\left(Q^{2} / Q_{0}^{2}\right)}, \pi=-\left(\left(11-\frac{2}{3} n\right) \frac{a_{s}\left(Q_{0}^{2}\right)}{4 \pi} \ln \left(0^{2} / Q_{0}^{2}\right)\right) \\
n=\text { number of flavars }
\end{aligned}
$$

Ath, however, effectively a plus sign in the denominator. As $Q \rightarrow-$, i.e. $r+0$, the copling becares waker, one has antishielding (in current slang, this plenomenon is called "asymptotic freedon"). It makes it possible to justify the soft potentiais corresponding to the observed levels (Figs 1, 2) of the quarkonia. We mantion in passing that in virtve of the guark spins and of the vector nature of the gluone co has the Elne stnuctures so dear to atomic physicists. 
This theorem, of rather fonal character, guarantees that one may (surming over the final states of any inclusive e.m. process) let the lepton mass $m_{1}$ tend to wero without creating terrible havoc.

\section{3 the Yorsie - Prautschx - suma relation}

This relation, similar in eagence to the old Bloch-Nordsieck theory, guarantees the absence of catastroghes (infra-red divergencies) in the limit $k \rightarrow 0$. Such a cutastrophe could be anticipated, but covicusly does not happen in, say, elastic electron scattering where the final state electron could radiate an infinite nuber of softer and softer photons.

From these three "pillars" and the "foundation" of local gauge invariance, one can derive - besides the innumerable atomic properties you are all faniliar with many inportant consequences. These are either interesting in themselves, or through the fact that they are readily ganeralized to strong interactions ( $Q C D)$. We discuss a few:

\subsubsection{Scale invariance at Iarge momentum transfer}

This means that in an inclusive reaction like

$$
e+\bar{e}+\gamma^{*}+\mu+\bar{\mu}+x
$$

where $X=$ any neutral state composed of leptons and photons

the cross section exhibits, to MOPT, a pointlike behaviour (thus scale invariance meaning that no lengths appear il: the fomulae):

$$
\begin{aligned}
& \alpha(e+\bar{e}+x)=\frac{4 \operatorname{ma}\left(Q^{2}\right)}{3 Q^{2}}\left(1+\frac{3}{4} \frac{a\left(Q^{2}\right)}{\pi}+c_{2}\left(\frac{a\left(Q^{2}\right)}{n}\right)+c_{1}\left(\frac{\alpha\left(Q^{2}\right)}{n}\right)^{3}+\cdots\right. \\
& \text { (valid for } \left.Q^{2}>\min _{\mu}^{2}\right) \text {. }
\end{aligned}
$$

Note the abeence of terms in in $m_{1}$, a consequence of the kN theorem. The raaction (3) is not one of purely acadenic interest. In fact, in é colliders the mon pair production is used in practice to monitor the luminosity of the machine,

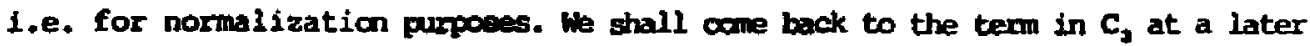
point.

It is interesting to replace the leptons in (3), either in the initial or the final state, by guarks. We thus consider 


$$
e+\ddot{e}+(q+\bar{q})+x
$$

and

$$
q+\bar{q}+\mu+\bar{\mu}+x
$$

The breckets in the first reaction regresent the fact that the quark and antigrark never appear as isolated physical particles in the final state. They can be proaned in a baind state (of spin-parity $7^{-}$equaling that of the $r$ ). Such pairs are precisely the 's quarkonia shown in Figs 1, 2. Their prabuction cross sections contain factars allowing for the fractional charges of the quarks and for theit "color". Process (5) represents mon pair production in the colliston of any two hadrons, to the extent that these contain (real or virtual) $\bar{q}$ 's. In the jargon it is called the "Drell-Yan" process; it has been the subject of much experimental investigation, and is one of our major sources of information about the quark "wavefunction" of hadrons.

Finally, one mey replace the leptons on both sides of Eq. (3) by guarks. Electromagretism than plays a subardinate role, so that the virtual photon f* has to be replaced by a vixtual gluon $g^{*}$. Thanks to the gauge structure comon to goD and $Q C D$, the essential results remain valid in the latter, with $a_{s}\left(Q^{3}\right)$ ceplacing a(d).

\subsection{2 saling and scaling violation at large monentum transfers}

("diseply inelastic" ecattering)

Consider (for pedagogical reascansf) the process

$$
\mu+e+\mu+e .
$$

One has for the differential cross section without radiation

$$
\frac{d o}{d \sigma}=\frac{m \alpha_{x}^{2}}{s} f(v), \quad(\sqrt{s}=c \cdot m \cdot \text { energy })
$$

which can be generalized to AOPT and to $Q D$ processes. Next consider, to please the tastes of atomic physicists, the inelastic scattering of electrons by muaniun

$$
e+(\mu \bar{\mu})+e^{\prime}+x
$$

Because of the inelasticity, one has now a doubly differential cross section, which can be written as

$$
\frac{d o^{2}}{d \alpha^{2}}=\left[\frac{d x}{d x}\right]_{\operatorname{en}} F(x)
$$


Where $x$ is the dimensianiess scaling variable

$$
x=\frac{\rho^{2}}{2 P+q} \cdot \frac{\text { (manentum transfer) }^{2}}{1 \text { (energy transfer) }}
$$

Wth $P$ the 4-momentun and $M$ the mass of the "Incident" avonlum. Equ. (10) is the basks of the parton model of deeply inelastic scattering of leptuns, where the ale of the wans in our "pedagogical" example is played by the guarks. The elastic collision between qark and lepton is turned into a (deeply) inelastic scattering of the lepton by the hadron, the final state $X$ consisting of real hadrons zather than free partons.

Because of the guxpe nature of QD, entirely similar arguments hold for partonparton collisions. Radiative corrections are, howerer, generally more important here, because a (s for strong I) is, at given $Q^{2}$. larger than $\alpha\left(Q^{2}\right)$ : gluons are mre easily radiated then photonsl Oansider resction (7) with photon radiation by the ingident mun. The differential cross section (8) is nodified as

$$
\frac{d g}{d s}=\frac{\pi \alpha_{I}^{2}}{s} f(u)\left[1+\frac{a}{n} \ln \frac{\rho^{2}}{m_{\mu}^{2}} \ln \Delta E / E\right]
$$

where $\Delta E / E$ is an experimental resolution. Sinilarly, the "structure function" $F(x)$ of the $\mu^{+} \mu^{-}$atan in Equ. (10) becomes

$$
F\left(x, \ln Q^{2} / Q_{0}^{2}\right)
$$

Thereby scale invariance is broken, although so explicit dependence on a length enters. Again, a logarithmic dependence as in (13) is taken over into rD. All structure functions "evalve", as was shown by Gribov and Lipatov, and by Altarelli and Parisi.

\subsubsection{Iow-eneroy theorem in Compton ecattering}

One can show that the forward scattering anglitude is given, as $\omega \rightarrow 0$, to AOPT for any epins by

$$
f(0)=-\frac{e^{2}}{m} \vec{a} \cdot \vec{E}-i \lim _{\mathrm{a}}^{2}(\vec{S} / \mathrm{s}) \cdot \vec{E} \cdot x \vec{E}+0\left(\omega^{2}\right) \text {. }
$$

where $\mu_{a}=\mu-e S / m$ defines the ancmalous moment for any spin. This relation, in combination with the cptical theorem, ensbles one to set limits on the composite scale of leplons. It also implies that the nomal $g$-factor $g=(\mu / s) /(e / 2 m)$ of any pointlike particle 1s 2. Indeed if the electron or muon were composite, 1.e. if

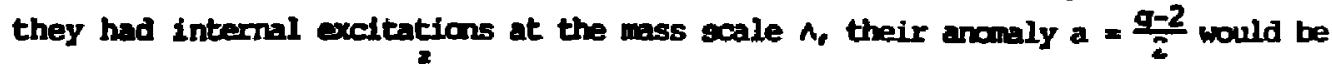
of ander $\left(\operatorname{mos}_{\mathrm{e}} / n\right)$ or $(\mathrm{n} / \mathrm{e} / \mathrm{n})^{2}$. 
The two cases depend whether ar not the interwctions of the underlying theory remerble gauge theories and conserve chiral invariance. In elther case, the present agreement between theory and experiment for the electron and mus anomalous wonts rules out an internal cale below $1 \mathrm{TeV}$, [ see e.g. S. J. Brodsky and J. Erimack, Ann. Fhys. 52, 315 (1969). S. J. Brodsky and S. D. Drell, Ehys. Rev. D22, 2256 (1920). 1

\subsubsection{Berocmalization of the wak angle $\theta_{-}$}

The standard theory of electroveak interactions contains two coupling constants but only one free parameter, the Neinberg angle $\theta_{w}$. The latter fixes the e.m. reak oconnection:

$$
e=g \sin \theta_{w}=g^{\prime} \cos \theta_{w}
$$

as well as the mass ratio of the two heavy gauge bocons:

$$
\text { m } / m_{2}=\cos \theta
$$

since e, i.e. $\alpha$, is a "running" coppling constant (see above), it is clear that $\theta_{w}$ Itself must be "running". These considerations are of interest for two reasons! (1) they will tell us at which energy e.m. and "weak" Interactions will become equally "strong", (11) by detemining ${ }_{w}$ at two energies, one can experifmentally verify the gauge nature of the theory.

\subsection{5 the Nambu-Bethe-salpeter (NBS) equation}

This covariant two-body equation, with wich this audience is certainiy familiar, allows to solve everything in principle, but little in actual practioe. This is for two reasons: (i) ane needs an infinite number of kernels, (ii) even in the Ladder approximation no analytic solution for 020 has been produced.

One interesting consequerce of the NBS equation is that by its reduction (in the case of two quarks) a scträdinger equation with a non-local potential energes.

see also comments below under "cpen problems".

\section{Oen problens ("the dirt")}

\subsection{Doss the perturtation series in goo converge?}

Nobody knows the answer, but pertaps there is no answer within the old classical framework, i.e. In a world nade of leptons and photons alone. Indeed charged leptons interact with each other by both $\mathrm{s}$ and $\mathrm{z}^{\circ}$ exchange, a fact alraady verified by experiment ( $\mu$-pair asyumet $:$, in è collisions). There are "grand" gchemes 
to unify electroweak and trong (000) focces, giving them equal strength at some very high (say $10^{\circ} \mathrm{GeV}$ ) enecgy. In such schenes the "tandau singularity" might be ared.

There exist acne exaiting umings from PT that the PT geries may not converge. Iet us mention two:

\subsubsection{The decay Inte of 's, positronium}

the current theoretical prealiction is

$$
r=\Gamma_{0}\left[1-10.282(\alpha / n)+\frac{1}{3}\left(a^{2} \ln \alpha\right)+(300 \pm 30)(\alpha / n)^{2}\right]
$$

The unexpectediy large coefficient of the last, experimentally determined term might well be the presage of rorse thing. to comel A similar behavior in $Q D D$, say in the analogous 3-gluen arribilation of $3, s_{2}$ charmonium, would be a real disaster, since $\alpha_{s}$ is larger than $\alpha$.

\section{1 .2 Radiative corrections to 000 Born cross section}

The inclusive cross-section for ete $\rightarrow$ hadrons is given by

$$
\left.0=0.11+\left(\frac{\alpha_{s}}{n}\right]+1.41\left(\frac{\alpha_{s}}{n}\right)^{2} f-64.809\left[\frac{\alpha_{s}}{n}\right]^{3}+\ldots\right]
$$

as reported by Corishny, Kataev and Larin (Dubna). This may be, if confirmed by independent calculations, an indication of the breakdown of the PT series in gauge theories.

\subsection{Proqress on the relativistic 2-boty equation}

There are three methods other than NBS. In the apprasch of Grotch and Yennie one uses an effective Dirac equation with non-local potentials derived from ei scattering. In a mare recent method, that of Caswell and Lepage, one starts from an effective Schradinger equation, egain with mon-local potentials. Eoth methods have been used to calculate higher oriar terms for $e p$, eè and eì atoms. A third approach, currently being used by S. Brodsky, T. Eller, H.C. Pauli and A. Tang, is that of "discretized light-cone quantization". These authors directly (i.e. numeelcally) diagonalize the light-cone Ismiltonian, of course with a trincated basis of Fock states. This yields both the mass spectrum (Levels) and the wave functions. The method works for any a, but results have only been reparted to date for $1+1$ dimersions. 
Acknowledgement SJB would like to acknowledge the support of the Alexander von Humboldt Foundation and the Max Planck Institute for Nuclear Physics, Heidelberg. 